\title{
Violation of treatment guidelines - hazard for rectal cancer patients
}

\author{
Eivind Jullumstro $\cdot$ Arne Wibe $\cdot$ Stian Lydersen • \\ Tom-Harald Edna
}

Accepted: 22 July 2011 / Published online: 23 August 2011

(C) The Author(s) 2011. This article is published with open access at Springerlink.com

\begin{abstract}
Aim The aim of this study was to evaluate temporal trends in treatment and outcome in rectal cancer diagnosed during 1980-2004 at Levanger Hospital.

Materials and methods A protocol for prospective registration of rectal cancer treated with total mesorectal excision including operative strategy, radiotherapy and surveillance was established at Levanger Hospital in 1980. In this study, all rectal cancer patients treated during 1980-2004 were included.
\end{abstract}

Eivind Jullumstrø died of cancer on 20 December 2010. We dedicate this article in thankful memory to him.

\section{E. Jullumstrø $\cdot$ T.-H. Edna}

Department of Surgery, Levanger Hospital,

Nord-Trøndelag Health Trust,

Levanger, Norway

A. Wibe

Department of Cancer Research and Molecular Medicine,

Unit for Gastroenterology,

Norwegian University of Science and Technology,

Trondheim, Norway

\section{A. Wibe}

Department of Surgery, St. Olavs Hospital,

Trondheim, Norway

\section{S. Lydersen • T.-H. Edna}

Department of Cancer Research and Molecular Medicine,

Unit for Applied Clinical Research,

Norwegian University of Science and Technology,

Trondheim, Norway

\section{T.-H. Edna $(\square)$}

Sykehuset Levanger,

Kirkegata 2,

7600 Levanger, Norway

e-mail: thedna@hnt.no
Results More patients received preoperative radiotherapy during 2000-2004, but otherwise there were no significant differences in presentation or treatment during 1980-2004. The 5 -year local recurrence rate after resection with curative intent was $4.5 \%(0-9.7), 18.7 \%(10.3-27.1)$ and $2.2 \%(0-6.7)$ in 1980-1989, 1990-1999 and 2000-2004 $(p=0.006)$, respectively. Out of a total of 23 cases of local recurrence, treatment guidelines, mainly with regard to radiotherapy, were violated in 19 cases. The 5-year overall survival after resection with curative intent was $65 \%$ (95\% confidence interval [CI] 55-76) during 1980-1989, 58\% (49-68) in 1990-1999 and 71\% (5983 ) in 2000-2004 (n.s). The 5-year relative survival was $83 \%$ (95\% CI 69-95) during 1980-1989, 71\% (59-81) in 19901999 and 84\% (69-98) in 2000-2004 (n.s).

Conclusion Rectal cancer patients experienced excellent outcomes in the period 1980-1989 and 2000-2004. Due to violations of treatment guidelines, the rate of local recurrence was much too high in the period 1990-1999. This article illustrates the importance of continuous quality assurance in the treatment of rectal cancer to maintain optimized outcomes for the patients.

Keywords Rectal cancer Guidelines · Quality assurance · Outcome

\section{Introduction}

Total mesorectal excision (TME) as described by Heald et al. in 1982 has [1] become the gold standard of rectal cancer surgery. When performed meticulously, it yields low rates of local recurrence (LR) and improved survival [1]. In Norway, a national rectal cancer project was launched in 1993 in order to improve outcome for rectal cancer patients. Of several interventions, TME was introduced as the 
preferred surgical technique and an educational programme, which included training courses and master classes, was implemented throughout Norway [2]. As a result, the risk of LR was decreased by $50 \%$ [3].

At Levanger Hospital, the modern principles of rectal cancer surgery were introduced in 1980, and a prospective protocol for operative strategy, radiotherapy and surveillance was established. Although excellent results were reported [4], an offer to participate in the TME educational programme was rejected by Levanger Hospital because it was a common belief among our staff that we had mastered this technique. However, the first biannual report from the Cancer Registry of Norway in 1997 revealed an unacceptably high rate of LR at Levanger hospital, and actions had to be taken. The protocol was regarded as sufficient and left unchanged, but the focus on adequate preoperative assessment to assign the right patients to neoadjuvant therapy was increased. In addition, the team of surgeons who performed TME surgery was strengthened by having specialists in colorectal surgery present during operation. In this study, a complete cohort of patients who were treated for rectal cancer at Levanger Hospital during 1980-2004 was analyzed to assess temporal trends in treatment and oncologic outcomes. We examined every case of LR in this time period in search of possible protocol violations.

\section{Patients and methods}

A complete cohort was assured by using data from the Norwegian Cancer Registry. The hospital health records of all patients treated for rectal cancer at Levanger Hospital from 1980 to the end of 2004 were reviewed. The patients were assigned into three separate periods, which depended on their date of surgery: 1980-1989, 1990-1999 and 20002004. The hospital served a defined patient catchment area with a population that increased slightly from 85,741 in 1980 to 88,858 in 2004 .

The upper limit for rectal cancer was defined as $15 \mathrm{~cm}$ from the anal verge measured on rigid proctoscopy. The surgery was performed by sharp dissection under visual guidance in the avascular plane surrounding the mesorectal fascia. A major resection with curative intent implied resection of the tumour bearing segment of rectum with no signs of metastases on preoperative investigations or by intraoperative examination, but included patients with microscopically involved margin and intraoperative perforations. The operation was considered curative if a microscopically free margin was confirmed and there was no bowel perforation. Circumferential resection margin (CRM) was defined as the shortest distance from the periphery of the tumour or tumour deposits to the resection margin. Residual tumour stage ( $\mathrm{R}$ stage) was registered where no residual tumour locally was classified as
R0 resection, microscopically involved margin as R1 resection and macroscopically residual tumour as $\mathrm{R} 2$ resection. To assign cancer stage we used the TNM Classification of Malignant Tumours, sixth edition [5]. A histological examination was missing in 17 patients, of which 12 had received best supportive care without any operation and five had received nonresective procedures.

The staff of specialist surgeons was stable and increased over the past 25 years. During the years 1990-1999, many undergraduates were responsible for the preoperative examinations, although they performed the surgical procedures together with a specialist in gastrointestinal surgery. The responsibility for treatment of rectal cancer was dispersed on more persons than during 1980-1989. Since 2000, rectal cancer surgery was no longer required for surgeons specializing in general surgery, and more dedicated teams were responsible for the preoperative examinations and the treatment.

During the operation, the routine was $5 \mathrm{~cm}$ distal margin for tumours in the upper and middle rectum, but a $2-\mathrm{cm}$ margin was accepted in lower tumours in order to avoid abdominoperineal resection.

The clinical follow-up programme after resection with curative intent was 5 years (range of follow-up 0-28.7 years), and the median follow-up with regard to survival was 9.4 years (range 5.0-28.7). The surveillance programme was principally the same for all 25 years, based on symptoms, clinical examination including proctoscopy, measure of carcinoembryonic antigen at every visit, chest radiography and colonoscopy at intervals. Liver ultrasound and CT scan were performed at intervals as these modalities became available.

LR was defined as recurrent disease in the pelvis or the perineum, regardless of whether it was distant metastasis or not. Mortality data were collected from the hospital patient administrative system. Postoperative mortality was defined as all deaths within 30 days after laparotomy or during the same hospital stay, regardless of time. Overall survival was estimated by inclusion of death from any cause. Relative survival was defined as the ratio of observed survival in rectal cancer patients to the expected survival of the general population of Norway.

\section{Perioperative radiotherapy and operative strategy}

Preoperative radiotherapy was recommended for patients who had been diagnosed with locally advanced tumours throughout the period, initially given as $46 \mathrm{~Gy}$, and in later years as 2 Gy $\times 25$ with concomitant tumour-sensitizing 5Fluorouracil. In 1980-1999, before the era of MRI, a tumour was considered locally advanced when it was fixed in the pelvis, or if it could not be moved in two planes at the preoperative examination. The surgery was performed according to the principles described by Bjerkeset and Edna [4]. Postoperative radiotherapy was recommended in cases of an R1 resection or perforation. 
Statistical methods

Two by two tables were analyzed using the unconditional $z$ pooled test, which is the unconditional version of Pearson's $\chi^{2}$ test [6]. The exact Cochran-Armitage test was used for testing trends in proportions. The medians of three samples were analyzed using the Kruskal-Wallis test. To analyze the association between the period of treatment as an explanatory variable and the dependent variables tumour stage, ASA score and the number of specialists in colorectal surgery, we used a proportional odds logistic regression model, also called ordinal logit model, which has been recommended by Agresti [7]. Kaplan-Meier survivor functions, with corresponding estimates and 95\% confidence intervals (CI), were calculated. The survival functions were compared using the log-rank test.

Relative survival was estimated using actuarial methods and analyzed with STATA [8]. Significance tests of excess mortality were done using a full likelihood approach. Norwegian population survival probabilities for every year from 1980, by sex and age, were downloaded from the Human Mortality Database [9]. Data were not available for 2009 , and according to standard practice, we made the assumption that the probabilities for 2009 were the same as for 2008 .

Two-sided $p$ values $<0.05$ were considered significant. The analyses were performed using SPSS 15.0, STATA 10.0 and StatXact 8.0.

The study was approved by the Regional Committee of Ethics and performed according to the Helsinki declaration.

\section{Results}

A total of 394 patients, 247 males and 147 females, were treated between 1980 and 2004. The median age at diagnosis was 69.9 years (range 37-90) during 19801989 and 70.1 years in 1990-1999 and 2000-2004 (range 35-93 and 44-91, respectively).

\section{Location}

A total of 125 tumours (32\%) were located $12-15 \mathrm{~cm}$ from the anal verge, $175(44 \%)$ were $6-11 \mathrm{~cm}$ from the anal verge and $94(24 \%)$ were $0-5 \mathrm{~cm}$ from the anal verge. The percentage of patients with a low rectal cancer was the same during the three periods studied.

\section{Presentation}

The type of presentation by time period is shown in Table 1 . The number of patients admitted as emergency cases with either obstruction or spontaneous tumour perforation was $4 \%(5 / 127)$ during $1980-1989,6 \%(10 / 177)$ in 1990-1999 and $4 \%(4 / 90)$ in 2000-2004. The stage at diagnosis was similar for all three periods (Table 2). Poor differentiation was reported in $13 \%$ of all rectal cancer cases in1980-1989 and $14 \%$ in both $1990-1999$ and 2000-2004.

\section{Treatment}

Type of treatment in relation to time period is shown in Table 3. Resection with curative intent was performed in $63.8 \%(81 / 127)$ of all rectal cancer cases during 1980-1989, $64.9 \%(115 / 177)$ in $1990-1999$, and $62.2 \%(56 / 90)$ in 2000 2004 (n.s.). For patients who had a resection with curative intent, radiotherapy was given preoperatively or postoperatively as presented in Table 4. Significantly more patients received preoperative radiotherapy during 2000-2004.

The rate of resections with curative intent performed with a specialist in colorectal surgery present increased significantly from the first to the last time periods (Table 5). A surgical trainee performed the operation in $37 \%(30 / 81)$ of all cases during 1980-1989, 44\% (51/115) in 1990-1999 and $12.5 \%(7 / 56)$ in $2000-2004(p=0.011)$.

The median operative time in operations with curative intent was 200 min (range 125-410) during 1980-1989, 165 min (84-370) during 1990-1999 and 150 min (67$310)$ during the last period $(p=0.002)$.

The median blood loss during operations with curative intent was 1,200 $\mathrm{ml}$ (300-9,000) during 1980-1989, $900 \mathrm{ml}(200-10,000)$ during 1990-1999 and $650 \mathrm{ml}(50$ $3,000)$ during the last period $(p<0.001)$.

The proportion with distal resection margin of $2 \mathrm{~cm}$ or more in operations with curative intent was $68 \%(55 / 81)$ during 1980-1989, 70\% (81/115) during 1990-1999 and $84 \%$ (47/56), during 2000-2004 ( $p=0.056)$.

Sphincter-sparing surgery was performed in $64 \%(52 / 81)$ of resections with curative intent during 1980-1989, 73\% $(84 / 115)$ in $1990-1999$ and $66 \%(37 / 56)$ in $2000-2004$.
Table 1 Type of presentation in relation to period $(\%)$

\begin{tabular}{lcccc}
\hline Type of presentation & $1980-1989$ & $1990-1999$ & $2000-2004$ & Total \\
\hline Obstruction without perforation & $0(0)$ & $7(4)$ & $2(2)$ & $9(2)$ \\
Spontaneous perforation & $5(4)$ & $3(2)$ & $2(2)$ & $10(3)$ \\
Elective presentation & $122(96)$ & $167(94)$ & $86(96)$ & $375(95)$ \\
Total & $127(100)$ & $177(100)$ & $90(100)$ & $394(100)$ \\
\hline
\end{tabular}


Table 2 Stage in relation to period (\%)

\begin{tabular}{lcccc}
\hline Stage & $1980-1989$ & $1990-1999$ & $2000-2004$ & \multicolumn{1}{l}{ Total } \\
\hline I & $26(21)$ & $25(14)$ & $16(18)$ & $67(17)$ \\
II & $32(25)$ & $53(30)$ & $23(26)$ & $108(27)$ \\
III & $32(25)$ & $44(25)$ & $19(21)$ & $95(24)$ \\
IV & $22(17)$ & $34(19)$ & $24(27)$ & $80(20)$ \\
Unknown & $15(12)$ & $21(12)$ & $8(9)$ & $44(11)$ \\
Total & $127(100)$ & $177(100)$ & $90(100)$ & $394(100)$ \\
\hline
\end{tabular}

Association between known stage and period of treatment: $\mathrm{OR}=1.17$ $(0.90-1.51), p=0.24$

Anastomotic leakage was diagnosed in 10\% (5/52) during $1980-1989,6 \%(5 / 84)$ in $1990-1999$ and $8 \%(3 / 37)$ in 2000-2004.

\section{Postoperative mortality}

After resection with curative intent, the postoperative mortality rate was $7.4 \%(6 / 81)$ during 1980-1989, 4.3\% $(5 / 115)$ in $1990-1999$ and $3.6 \%(2 / 56)$ in $2000-2004(p=$ $0.34)$. For patients treated with palliative intent, the corresponding numbers were $8.3 \%(2 / 24), 19.4 \%(7 / 36)$ and $0 \%(0 / 20)$. The overall postoperative mortality was $24 \%$ in patients presenting with spontaneous perforation or bowel obstruction.

\section{Local recurrence}

The 5-year estimated LR rate after resection with curative intent was 4.5\% (0-9.7), 18.7\% (10.3-27.1) and 2.2\% (0$6.7)$ in 1980-1989, 1990-1999 and 2000-2004 ( $p=0.006)$, respectively. Out of 11 resections with curative intent, classified as R1 resections, ten patients developed LR (Table 6). After curative resection with a distal clearance of less than $2 \mathrm{~cm}$, LR developed in 11\% (10/92) compared to only $3 \%(4 / 138)$ when the distal clearance was $>2 \mathrm{~cm}(p=$
0.014). Out of ten patients with $\mathrm{R} 1$ resections and four patients with intraoperative tumour perforation who later developed LR, only one patient received postoperative RT. Radiotherapy was only given to one of the 23 patients who later developed an LR. When no obvious risk factor was present (T1-3 cancer, no perforation, no $\mathrm{R} 1$ resection or distal clearance $>2 \mathrm{~cm}$ ), only four patients developed an LR.

Long-term survival

For all stages together, the estimated 5-year overall survival was 48\% (95\% CI 40-58) during 1980-1989, 40\% (33-48) in 1990-1999 and 50\% (40-61) in 2000-2004 (n.s). The corresponding estimated 5-year overall survival after resection with curative intent was 65\% (95\% CI 55-76) during 1980-1989, 58\% (49-68) in 1990-1999 and 71\% (59-83) in 2000-2004 (n.s). For all stages together, the estimated 5-year relative survival was 63\% (95\% CI 51-73) during 1980-1989, 50\% (41-58) in 1990-1999 and 59\% (46-71) in 2000-2004 (n.s.). The corresponding estimated 5 -year relative survival after resection with curative intent was 83\% (95\% CI 69-95) during 1980-1989, 71\% (59-81) in 1990-1999 and 84\% (68-97) in 2000-2004 (n.s).

\section{Discussion}

Excellent overall survival, relative survival and LR rates were achieved in 1980-1989 and 2000-2004. However, during 1990-1999, the LR rate was unacceptably high and survival was correspondingly low. In almost all cases of LR, violation of treatment guidelines could be identified.

This paper presents one of the longest running experiences with TME for rectal cancer. A complete cohort of patients was obtained, thereby avoiding selection bias. A protocol for treatment and surveillance was established in 1980 and was unchanged throughout the study period.

Table 3 Type of treatment in relation to period (\%)

\begin{tabular}{|c|c|c|c|c|}
\hline Type of treatment & $1980-1989$ & 1990-1999 & 2000-2004 & Total \\
\hline Best supportive care, no operation & $12(9)$ & $22(12)$ & $10(11)$ & $44(11)$ \\
\hline Nonresective procedure: Stoma, bypass, explorative laparotomy, or laparoscopy & $7(6)$ & $14(8)$ & $9(10)$ & $30(8)$ \\
\hline Palliative resections & $17(13)$ & $22(12)$ & $11(12)$ & $50(13)$ \\
\hline Local resection (trans-anal/endoscopic) & $10(8)$ & $4(2)$ & $4(4)$ & $18(5)$ \\
\hline \multicolumn{5}{|l|}{ Major resection with curative intent } \\
\hline Involved CRM without bowel perforation & $0(0)$ & $8(5)$ & $0(0)$ & $8(2)$ \\
\hline $\mathrm{R} 0$ resection and bowel or tumour perforation & $4(3)$ & $5(3)$ & $2(2)$ & $11(3)$ \\
\hline Involved CRM and perforation & $2(2)$ & $1(1)$ & $0(0)$ & $3(1)$ \\
\hline Curative resection & $75(59)$ & $101(57)$ & $54(60)$ & $230(58)$ \\
\hline Total & $127(100)$ & $177(100)$ & $90(100)$ & $394(100)$ \\
\hline
\end{tabular}


Table 4 Radiotherapy for patients treated with curative intent $(\%)$

$p=0.008$ (Cochran-Armitage trend test of radiotherapy vs. no radiotherapy)

\begin{tabular}{lcccc}
\hline & $1980-1989$ & $1990-1999$ & $2000-2004$ & Total \\
\hline No radiotherapy & $73(90.1)$ & $111(96.5)$ & $41(73.2)$ & $225(89.3)$ \\
Preoperative radiotherapy & $5(6.2)$ & $1(0.9)$ & $12(21.4)$ & $18(7.1)$ \\
Postoperative radiotherapy & $2(2.5)$ & $3(2.6)$ & $3(5.4)$ & $8(3.2)$ \\
Preoperative and postoperative radiotherapy & $1(1.2)$ & $0(0)$ & $0(0)$ & $1(0.4)$ \\
Total & $81(100)$ & $115(100)$ & $56(100)$ & $252(100)$ \\
\hline
\end{tabular}

Although the protocol was implemented for prospective registration, this study still has the limitations of a retrospective study in that the data were analyzed retrospectively. This is a minor concern considering that overall survival and relative survival are robust parameters [10], although this might have led to underestimation of LR rates. For instance, old and fragile patients received less follow-up, which could result in LR being undisclosed. However, half of all cases with LR were diagnosed in patients older than 75 years.

The results were significantly worse during 1990-1999, as can be seen by the estimated 5-year LR rates: $4.5 \%$ during $1980-1989,18.7 \%$ in $1990-1999$ and $2.2 \%$ in 2000-2004 after resections with curative intent. In order to understand why LR occurred, all cases of LR were analyzed with particular emphasis on whether the protocol outlined in 1980 had been followed. Preoperative radiotherapy was recommended in locally advanced cases to achieve R0 resections. However, none of the four patients with a stage T4 cancer who later developed LR received preoperative radiotherapy. During 2000-2004, significantly more patients received preoperative radiotherapy, and excellent results concerning LR were achieved.

The CRM is a strong predictor of LR, distant metastasis and survival [11-13] in rectal cancer. The main reason why TME has been so successful in lowering LR rates compared to traditional rectal resections is its ability to achieve $\mathrm{R} 0$ resections [14]. In a recent publication [15], a CRM $<2 \mathrm{~mm}$ was associated with poorer prognosis. In this study, we found that in a total of $11 \mathrm{R} 1$ resections with curative intent, out of which nine where operated on during 1990-1999, ten patients later developed LR. Only one of these ten patients received postoperative radiotherapy. Although recommended in our protocol, Marijnen et al. [16] found little evidence to support postoperative radiotherapy as this was of no benefit for patients receiving non-radical resections.
On the other hand, preoperative radiotherapy was effective in cases of narrow margins (1.1-2 $\mathrm{mm}$ ) and wider margins. Knowing this, selecting patients at risk for $\mathrm{R} 1$ resections for preoperative radiotherapy to achieve $\mathrm{R} 0$ resections is far more important than offering postoperative radiotherapy to patients with microscopically involved margin.

Intraoperative perforation during resection of rectal cancer increases the LR rate and reduces survival [17, 18]. Although this remains to be proven effective in this setting [17], postoperative radiotherapy was recommended in our protocol and has since been implemented in both Norwegian and American guidelines [19, 20]. There is no upper age limit for adjuvant radiotherapy in Norway, but individual considerations must be taken for those aged above 75 years as radiotherapy substantially increases the risk of death from causes unrelated to rectal cancer in this age group [21]. Out of four patients with tumour perforation, three did not receive postoperative radiotherapy, all aged above 80 years.

Although there is conflicting evidence concerning how long the distal margin should be in resections of rectal cancers, many researchers [20,22] recommend a distal clearance of $>2 \mathrm{~cm}$ in $\mathrm{AR}$, which is recommended in our protocol. The rationale underlying this is the finding that intramural spread from rectal cancers $>1 \mathrm{~cm}$ from the primary lesion is uncommon [23] and even more so if the patient has been subject to preoperative RT [24]. In the present study, significantly more patients developed LR with a distal clearance of less than $2 \mathrm{~cm}$. Furthermore, none of these patients had received neoadjuvant RT. In a total of 15 patients with distal clearance $<2 \mathrm{~cm}$ and LR, 13 underwent operation during 1990-1999. In surgery of low rectal cancers, there is often a dilemma concerning oncologic radicality and the avoidance of stomas. This study supports the view that a distal clearance of at least $2 \mathrm{~cm}$ should be given priority and in cases where this is
Table 5 Number of specialist surgeons (\%) attending resections with curative intent

Association between number of specialist surgeons and period of treatment: $\mathrm{OR}=7.7(95 \% \mathrm{CI}$ 4.7-12.9), $p<0.001$

\begin{tabular}{lcccc}
\hline Number of specialist surgeons & $1980-1989$ & $1990-1999$ & $2000-2004$ & Total \\
\hline 0 & $17(21)$ & $1(0.9)$ & $0(0)$ & $18(17.1)$ \\
1 & $60(74.1)$ & $91(79.1)$ & $20(35.7)$ & $171(67.9)$ \\
$2-3$ & $4(4.9)$ & $23(20)$ & $36(64.3)$ & $63(25)$ \\
Total & $81(100)$ & $115(100)$ & $56(100)$ & $252(100)$ \\
\hline
\end{tabular}


Table 6 Operative characteristics and radiotherapy in patients with local recurrence after resection with curative intent

\begin{tabular}{|c|c|c|c|c|c|c|c|c|c|c|}
\hline $\begin{array}{l}\text { Sex and } \\
\text { age }\end{array}$ & Year & T-stage & + Nodes & $\mathrm{CRM}>2 \mathrm{~mm}$ & $\begin{array}{l}\text { Level of } \\
\text { tumour }\end{array}$ & $\begin{array}{l}\text { Distal } \\
\text { margin }>2 \mathrm{~cm}\end{array}$ & $\mathrm{R}$ status & $\begin{array}{l}\text { Perforation } \\
\text { of tumour }\end{array}$ & $\begin{array}{l}\text { Preoperative } \\
\text { radiotherapy }\end{array}$ & $\begin{array}{l}\text { Postoperative } \\
\text { radiotherapy }\end{array}$ \\
\hline ㅇ 84 & 1984 & 4 & $\mathrm{Y}$ & $\mathrm{N}$ & Middle & $\mathrm{Y}$ & 1 & $\mathrm{Y}$ & $\mathrm{N}$ & $\mathrm{N}$ \\
\hline 오 58 & 1984 & 4 & $\mathrm{Y}$ & $\mathrm{N}$ & Middle & $\mathrm{Y}$ & 1 & $\mathrm{Y}$ & $\mathrm{N}$ & $\mathrm{Y}$ \\
\hline ㅇ 83 & 1985 & 3 & $\mathrm{Y}$ & UK & Lower & $\mathrm{Y}$ & 0 & $\mathrm{~N}$ & $\mathrm{~N}$ & $\mathrm{~N}$ \\
\hline ๙ 62 & 1989 & 2 & $\mathrm{~N}$ & UK & Middle & $\mathrm{N}$ & 0 & $\mathrm{~N}$ & $\mathrm{~N}$ & $\mathrm{~N}$ \\
\hline ㅇ 75 & 1990 & 3 & $\mathrm{Y}$ & UK & Middle & $\mathrm{N}$ & 0 & $\mathrm{~N}$ & $\mathrm{~N}$ & $\mathrm{~N}$ \\
\hline ठิ 72 & 1991 & 3 & $\mathrm{~N}$ & UK & Upper & $\mathrm{N}$ & 0 & $\mathrm{~N}$ & $\mathrm{~N}$ & $\mathrm{~N}$ \\
\hline 우 73 & 1991 & 3 & $\mathrm{~N}$ & UK & Lower & $\mathrm{Y}$ & 0 & $\mathrm{~N}$ & $\mathrm{~N}$ & $\mathrm{~N}$ \\
\hline q 79 & 1991 & 4 & $\mathrm{Y}$ & $\mathrm{N}$ & Middle & Y & 1 & $\mathrm{~N}$ & $\mathrm{~N}$ & $\mathrm{~N}$ \\
\hline ठิ 74 & 1992 & 3 & $\mathrm{~N}$ & UK & Middle & $\mathrm{N}$ & 0 & $\mathrm{~N}$ & $\mathrm{~N}$ & $\mathrm{~N}$ \\
\hline ふ 72 & 1993 & 3 & $\mathrm{Y}$ & $\mathrm{N}$ & Upper & $\mathrm{N}$ & 1 & $\mathrm{~N}$ & $\mathrm{~N}$ & $\mathrm{~N}$ \\
\hline q 70 & 1993 & 2 & $\mathrm{~N}$ & Y & Middle & $\mathrm{N}$ & 0 & $\mathrm{~N}$ & $\mathrm{~N}$ & $\mathrm{~N}$ \\
\hline o 76 & 1993 & 3 & $\mathrm{Y}$ & $\mathrm{N}$ & Upper & $\mathrm{Y}$ & 1 & $\mathrm{~N}$ & $\mathrm{~N}$ & $\mathrm{~N}$ \\
\hline วิ73 & 1994 & 3 & $\mathrm{~N}$ & $\mathrm{~N}$ & Middle & $\mathrm{N}$ & 1 & $\mathrm{~N}$ & $\mathrm{~N}$ & $\mathrm{~N}$ \\
\hline วิ 81 & 1995 & 1 & $\mathrm{~N}$ & UK & Upper & $\mathrm{N}$ & 0 & $\mathrm{Y}$ & $\mathrm{N}$ & $\mathrm{N}$ \\
\hline ふ) 52 & 1997 & 3 & $\mathrm{~N}$ & $\mathrm{~N}$ & Upper & $\mathrm{N}$ & 1 & $\mathrm{~N}$ & $\mathrm{~N}$ & $\mathrm{~N}$ \\
\hline q 79 & 1997 & 4 & $\mathrm{Y}$ & $\mathrm{N}$ & Lower & $\mathrm{N}$ & 1 & $\mathrm{~N}$ & $\mathrm{~N}$ & $\mathrm{~N}$ \\
\hline כ) 84 & 1998 & 3 & $\mathrm{Y}$ & Y & Lower & Y & 0 & $\mathrm{~N}$ & $\mathrm{~N}$ & $\mathrm{~N}$ \\
\hline ㅇ 62 & 1998 & 3 & $\mathrm{Y}$ & Y & Middle & Y & 0 & $\mathrm{~N}$ & $\mathrm{~N}$ & $\mathrm{~N}$ \\
\hline ภิ 59 & 1999 & 2 & $\mathrm{~N}$ & UK & Middle & $\mathrm{N}$ & 0 & $\mathrm{~N}$ & $\mathrm{~N}$ & $\mathrm{~N}$ \\
\hline ふ 75 & 1999 & 3 & $\mathrm{~N}$ & $\mathrm{~N}$ & Middle & $\mathrm{N}$ & 1 & $\mathrm{~N}$ & $\mathrm{~N}$ & $\mathrm{~N}$ \\
\hline ふ) 74 & 1999 & 3 & $\mathrm{Y}$ & Y & Middle & $\mathrm{N}$ & 0 & $\mathrm{~N}$ & $\mathrm{~N}$ & $\mathrm{~N}$ \\
\hline o 78 & 1999 & 3 & $\mathrm{~N}$ & $\mathrm{~N}$ & Middle & $\mathrm{N}$ & 1 & $\mathrm{~N}$ & $\mathrm{~N}$ & $\mathrm{~N}$ \\
\hline วิ 85 & 2003 & 3 & $\mathrm{Y}$ & $\mathrm{Y}$ & Upper & $\mathrm{N}$ & 0 & $\mathrm{Y}$ & $\mathrm{N}$ & $\mathrm{N}$ \\
\hline
\end{tabular}

Level of tumour - distance from the anal verge: $12-15 \mathrm{~cm}$, upper rectum; $6-11 \mathrm{~cm}$, middle rectum, $<6 \mathrm{~cm}$, lower rectum

$Y$ yes, $N$ no, $U K$ unknown

hard to achieve due to sphincter-preserving surgery, preoperative RT should be considered.

The number of specialists in colorectal surgery present at operation increased during the years of the present study. After the year 2000, rectal cancer surgery was no longer required for surgeons who are specialists in general surgery in Norway, and the number of operations performed by a trainee was significantly reduced. The current policy at Levanger Hospital is that rectal cancer resections should be performed by or under the supervision of a specialist surgeon and having two specialists present is recommended. Borowski et al. [25] found no difference in anastomotic leak rates, operative mortality or survival between unsupervised trainees, supervised trainees and consultants. We still believe that the introduction of more competent teams performing the surgery has contributed to better results during 2000-2004. The better results during 1980-1989 compared to 19901999 are perhaps dependent on there being far more experienced trainees working at the hospital during the first period. During the 1980s, the trainees were more experienced in general surgery (6-11 years experience) than the surgeons in the 1990s (3-6 years experience).
Preoperative radiotherapy at biologically effective doses $\geq 30$ Gy has been shown to reduce the risk of LR and death from rectal cancer, and postoperative radiotherapy has been shown to reduce the risk of LR [21]. Preoperative chemoradiation is even more effective in lowering the LR rate, and an additional effect on survival is possible [26]. At Levanger Hospital, adjuvant radiotherapy has been recommended since 1980, but was barely used during 1990 1999, which may explain why the rate of LR was high and survival correspondingly low during these years. For the years 2000-2004, when the LR rate and survival were excellent, $21 \%$ received preoperative radiotherapy. If the quality of surgery performed was evaluated by operative time, blood loss, the proportion with sphincter-sparing surgery, by the proportion without postoperative anastomotic leakage and by the proportion with a resection margin of at least $2 \mathrm{~cm}$, the quality of the surgery performed for curative intent did not seem to be inferior during 1990 1999 compared with 1980-1989. Even if experienced surgeons assisted the many surgical trainees during the operations, and the operations were adequate, the ultimate outcome was inferior for some patients during 1990-1999, 
and this was probably mainly due to a lack of referral for radiotherapy. In many cases, the preoperative judgment concerning radiotherapy failed during 1990-1999. From year 2000, dedicated teams took care of preoperative evaluation and surgery, and the introduction of preoperative MRI for all patients with cancer of the rectum from this time on, made it possible with a much better and objective selection of those who needed preoperative radiotherapy.

In the present study, the estimated 5-year overall survival after resection with curative intent was $65 \%$ (95\% CI 55-76) during 1980-1989, 58\% (49-68) in 1990-1999 and 71\% (5983) in 2000-2004 (n.s). A higher rate of LR during 19901999 was accompanied by a lower survival rate. The negative prognostic effect of LR on survival is well documented [2].

\section{Conclusions}

Excellent results were seen during the 1980s due to the implementation of modern principles of rectal cancer treatment at Levanger Hospital in 1980. However, reports from the national rectal cancer registry revealed poor results for patients treated in Levanger during the 1990s, and the current paper discloses that violations of the treatment guidelines, particularly with respect to radiotherapy for patients with advanced stages, had serious effects on patient prognosis during these years. Actions were taken to improve compliance regarding treatment guidelines for rectal cancer and to strengthen the surgical team which took care of the patients preoperatively as well as performed the TME surgery. During 2000-2004, the results were once again excellent. The present study illustrates that although treatment guidelines and surgical technique may be adequate, continuous focus on quality assurance and the collective efforts of the members of the multidisciplinary team are mandatory to maintain optimized outcomes for rectal cancer patients.

Open Access This article is distributed under the terms of the Creative Commons Attribution Noncommercial License which permits any noncommercial use, distribution, and reproduction in any medium, provided the original author(s) and source are credited.

\section{References}

1. Heald RJ, Husband EM, Ryall RD (1982) The mesorectum in rectal cancer surgery - the clue to pelvic recurrence? Br J Surg 69 (10):613-616

2. Wibe A, Moller B, Norstein J et al (2002) A national strategic change in treatment policy for rectal cancer-implementation of total mesorectal excision as routine treatment in Norway. A national audit. Dis Colon Rectum 45(7):857-866

3. Wibe A, Carlsen E, Dahl O et al (2006) Nationwide quality assurance of rectal cancer treatment. Colorectal Dis 8(3):224-229
4. Bjerkeset T, Edna TH (1996) Rectal cancer: the influence of type of operation on local recurrence and survival. Eur J Surg 162 (8):643-648

5. Sobin L, Wittekind C (2002) TNM classification of malignant tumors. John Wiley, New York

6. Lydersen S, Fagerland MW, Laake P (2009) Recommended tests for association in $2 \times 2$ tables. Stat Med 28(7):1159-1175

7. Agresti A (2007) An introduction to categorical data analysis. Wiley-Interscience, Hoboken

8. Dickman PW, Sloggett A, Hills M, Hakulinen T (2004) Regression models for relative survival. Stat Med 23(1):51-64

9. Human Mortality Database. http://www.mortality.org/(2008)

10. Platell CF, Semmens JB (2004) Review of survival curves for colorectal cancer. Dis Colon Rectum 47(12):2070-2075

11. Nagtegaal ID, Marijnen CA, Kranenbarg EK, Van De Velde CJ, van Krieken JH (2002) Circumferential margin involvement is still an important predictor of local recurrence in rectal carcinoma: not one millimeter but two millimeters is the limit. Am J Surg Pathol 26(3):350-357

12. Quirke P, Durdey P, Dixon MF, Williams NS (1986) Local recurrence of rectal adenocarcinoma due to inadequate surgical resection. Histopathological study of lateral tumour spread and surgical excision. Lancet 2(8514):996-999

13. Wibe A, Rendedal PR, Svensson E et al (2002) Prognostic significance of the circumferential resection margin following total mesorectal excision for rectal cancer. Br J Surg 89(3):327-334

14. Heald RJ, Moran BJ, Ryall RD, Sexton R, MacFarlane JK (1998) Rectal cancer: the Basingstoke experience of total mesorectal excision, 1978-1997. Arch Surg 133(8):894-899

15. Bernstein TE, Endreseth BH, Romundstad P, Wibe A (2009) Circumferential resection margin as a prognostic factor in rectal cancer. Br J Surg 96(11):1348-1357

16. Marijnen CA, Nagtegaal ID, Kapiteijn E et al (2003) Radiotherapy does not compensate for positive resection margins in rectal cancer patients: report of a multicenter randomized trial. Int $\mathrm{J}$ Radiat Oncol Biol Phys 55(5):1311-1320

17. Eriksen MT, Wibe A, Syse A, Haffner J, Wiig JN (2004) Inadvertent perforation during rectal cancer resection in Norway. Br J Surg 91(2):210-216

18. Jorgren F, Johansson R, Damber L, Lindmark G (2010) Oncological outcome after incidental perforation in radical rectal cancer surgery. Int J Colorectal Dis 25(6):731-740

19. Norwegian Gastro Intestinal Group (2008) Guidelines for Colorectal and Anal Cancer

20. Tjandra JJ, Kilkenny JW, Buie WD et al (2005) Practice parameters for the management of rectal cancer (revised). Dis Colon Rectum 48(3):411-423

21. Colorectal Cancer Collaborative Group (2001) Adjuvant radiotherapy for rectal cancer: a systematic overview of 8,507 patients from 22 randomised trials. Lancet 358(9290):1291-1304

22. Wasserberg N, Gutman H (2008) Resection margins in modern rectal cancer surgery. J Surg Oncol 98(8):611-615

23. Kwok SP, Lau WY, Leung KL, Liew CT, Li AK (1996) Prospective analysis of the distal margin of clearance in anterior resection for rectal carcinoma. Br J Surg 83(7):969-972

24. Mezhir JJ, Smith KD, Fichera A et al (2005) Presence of distal intramural spread after preoperative combined-modality therapy for adenocarcinoma of the rectum: what is now the appropriate distal resection margin? Surgery 138(4):658-663

25. Borowski DW, Ratcliffe AA, Bharathan B et al (2008) Involvement of surgical trainees in surgery for colorectal cancer and their effect on outcome. Colorectal Dis 10(8):837-845

26. Ceelen W, Fierens K, Van NY, Pattyn P (2009) Preoperative chemoradiation versus radiation alone for stage II and III resectable rectal cancer: a systematic review and meta-analysis. Int J Cancer 124(12):2966-2972 